\title{
Realization of wire selection platform based on three dimensional visualization technology
}

\author{
Peng Huang, Rui Liu, Zhenggang Fang and Hongbing Shen \\ China Electric Power Research Institute, China
}

\begin{abstract}
The three dimensional wire selection platform of the power transmission line is constructed by modelling technology and three dimensional scene render technology in combination with characteristics of the digital elevation, the digital ortho image and the earth geography information system. Through application of design and wire selection in some $330 \mathrm{kV}$ I and II circuit line engineering, it shows the design technical person can see scene of the three dimensional power transmission line clearly and livingly when the three dimensional visualization technology is applied in the optimized wire selection practice of the power transmission line, optimizes route of the line, saves investment of the line, reduces labour strength of person and finally improves design quality of the line.
\end{abstract}

\section{Introduction}

Route selection and investigation are extremely important key loops during the power transmission line design process, reasonability of the wire selection plan plays an important function on economic index, technical index and construction, operation conditions of the line. Selection of the power transmission line takes the space geographical information as basis. The traditional wire selection method takes the middle and small scale topography map as basis for rough selection, which realizes refinement and optimization of the plan through field investigation and measurement treatment on basis of rough selection. Because the current 1:10000 and 1:50000 topology maps applied in our country are mostly measured in 1980s or early, which are very obsolete and can' $t$ reflect current ground status during design[1]. At same time the topology map isn't intuitive and size of the map is limited, the design person only find the optimum line within the scope wide $30 \mathrm{~km}$ along the line. It is difficult for the traditional wire selection method to select the ideal route plan, which can't meet demand of power construction. How to overcome disadvantage of wire selection in the power transmission map in the topography map is a problem which is paid attention by the power designer in our country.

Following development of the modern investigation and measurement technology and appearance of the versatile business investigation and measurement image data, we are easy to obtain the demanded data. If the three dimensional visualization technology is applied in the wire selection of the power transmission line, when the investigation and measurement designer sees the ground model in the computer, it is convenient and intuitive as seeing ground from the air, which can optimize the routine plan within the large scope, it can see the relative relationship between the line and various buildings, the road and the railway etc, and obtain the effect which is difficult to obtain by the traditional wire selection method and reduce labour strength of the investigation and measurement design person[2].

\section{Three dimensional visualization technology}

At present, the three dimensional visualization technology is gradually applied in every field of the society and the living, such as digital city construction, military application, environment monitoring, scene spot planning, geography and mine activity, hydro geologic activity, traffic monitoring, estate development, medical aid etc. aspects. Appearance of OSG based on the OpenGL technology etc. three dimensional display platform, Quest 3D, Virtual tools, Skyline based on the DirectX 3D technology etc. system platforms greatly pushes forward development of the three dimensional visualization simulation and virtual technology.

Three dimensional wire selection of the power transmission line realizes effective mixture of the digital elevation model, the digital ortho image, the three dimension data and other GIS data information based on the three dimensional GIS platform, which re-appears real condition in the power construction environment of the power transmission line.

\subsection{Geographic information system}

Geographic Information System is an important tool to obtain, process, manage and analyze Geographic space d- 
ata. Function mainly includes data input and edit, data management, data processing, data display and output etc.

The three dimensional GIS platform can realize integration expression of the ground, earth surface, underground, under water information, which solves seamless integration of the three dimensional CAD design achievement and the three dimensional space information platform, two dimensional and three dimensional integration etc problem. The EV-Globe platform integrates the latest geographic information and the three dimensional software technology, which has the large scope, massive, multiple source data integration management and quick three dimension real-time roaming function, it supports inquiry, analysis and calculation of the three dimensional space, it can integrate with the traditional GIS software and provides the fundamental image documents within the global scope, it can construct the three dimensional space information service system conveniently and quickly, which can complete expansion from the two dimensional GIS system to the three dimensional system quickly, it is a new generation of the large space information service platform.

\subsection{Establishment of three dimensional model}

\subsubsection{Parameterization modelling technology}

Parameterization modelling technology is a technology which controls size and information of the model through parameter data. Main characteristic is to apply size driving, i.e., size of the parameterization model is expressed by the corresponding relationship; it isn't expressed by the determined value. When one parameter value is changed, all sizes related to it will be changed automatically, and a new same type of the model will be generated.

The user will realize modification of the model through modifying the size, which will generate a part model series with same shape and different specification. Parameterization model expresses the geometrical graph as the geometrical restraint model which consists of the geometrical element and its restraint relationship through seizing the restraint relationship between the geometrical elements in the model. The key in the parameterization modelling is to establish the geometrical restraint relationship, i.e., topology restraint and size restraint. Topology restraint is quantitative description on structure of the product, it expresses relationship between the geometrical element topology and the structure, such as parallelism, symmetry and verticality etc. These relationships maintain unchanged during size driving process of the graph. Size restraint expresses position relationship between the geometrical element through the restraint expressed by the size indication, such as distance size, angle size and radius size etc, is shown in fig 1 . It is the object of parameterization driving. Its features are to realize serialization design through modifying size of the graph on basis maintaining topology relationship of the original graph unchanged.

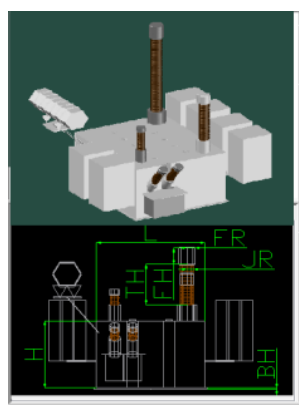

Figure 1. Parameterization modelling sample of transformer.

\subsubsection{Graphic modelling technology}

Graphic modelling technology is an important part during construction of the three dimensional model. Some equipment with complicated and versatile shape can realize model construction through the parameterization modelling. The graphic modelling way can make up disadvantage of the parameterization modelling, which becomes an essential model construction way. Secondly, when parameterization modelling can't realize high precision drawing of the complicate servant of some equipment and simulation requirements on the equipment are very high, it is only realized through the graphic modelling technology.

The model which is constituted by the graphic modelling technology shall have the corresponding property parameters, which can include two types of the parameter information. One is geometrical parameters, which can record space relationship of the nodes in the three dimension model, such as the appearance size, the diameter of the inner hole etc relevant parameters of the three dimensional model; another is electrical parameters, which can record the electrical property parameters of the three dimensional model, such as type of the equipment, material information etc relevant parameters, is shown in fig 2 .

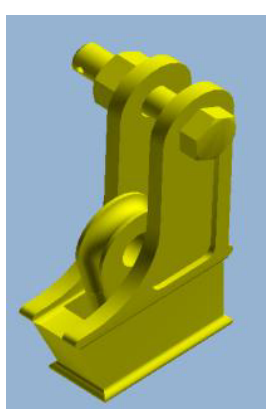

Figure 2. Sample of graphic modelling.

\subsection{Construction of power transmission line scene}

Optimization design of the graphic rendering algorithm is carried out as three parts during large scene rendering process of the power transmission line, including rendering of terrain and topology three dimensional scene model, rendering of the crossing object three dimensional model and rendering of the electrical equipment model[35]. 


\subsubsection{Rendering of terrain and topology three dimensional scene model}

Geographical information data for rendering of terrain and topology consists of the DEM elevation data and the DOM video data. The method to reduce consumption of a large quantity of data on the memory etc hardware device resource is to apply the blocking and grading rendering technology to solve visualization issue of the field. In order to realize switching of the geographic information data in different grade and different block quickly, the pyramid model and the four fork tree structure are applied during pre-processing and memory of the original geographic information data, is shown in fig 3 .


Figure 3. Processing flow of DEM and DOM data.

\subsubsection{Rendering of the crossing object three dimensional model and electrical equipment model}

Besides the geological information data in the three dimension simulation scene, data of the additional object on the floor are massive. It includes the crossing object (such as the house, the road, the power line etc) and the equipment in the power transmission line (such as the power transmission tower and the fitting etc). Geographical information data and these additional models constitute the complete three dimension simulation scene of the power transmission grid engineering. The rendering efficiency of the large scene scope and the detail effect of the model shall be considered during rendering of the model.

The rendering of the model is processed by the sectional loading, grading display method. Carry out sectional loading of the model in the whole three dimensional scene through position distance of the viewing angle and scope of the flat section head scope. The same model can be controlled precisely through observing the distance of the viewing angle. Only the low precision model and the outer profile frame of the model are rendered during long distance display. When observation distance is too short, load and render the finest detail characteristic of the model, the visualization display in the same scene can be realized in the large scale of the model by this way, is shown in fig 4 .
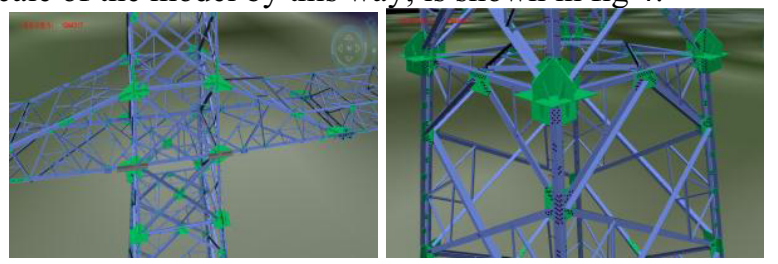

Figure 4. Fineness rendering sample of model

\section{Engineering application conditions}

\subsection{Engineering general}

For the newly built $330 \mathrm{kV}$ I, II circuits line engineering, the whole line consists of two single circuits which are parallel set up. The whole length of the preliminary design route is $72.5 \mathrm{~km}$; terrain unit along the line takes the aggraded valley plain, desert as primary, is shown in table 1 .

Table 1. Table of route length and terrain distribution condition

\begin{tabular}{|c|c|c|c|}
\hline Full name & $\begin{array}{c}\text { Altitude } \\
(\mathbf{m})\end{array}$ & $\begin{array}{c}\text { Route length } \\
(\mathbf{k m})\end{array}$ & $\begin{array}{c}\text { Optimized } \\
\text { adjustment } \\
\text { plan }\end{array}$ \\
\hline Gebi plain & $1270 \sim 1850$ & 61.3 & $84 \%$ \\
\hline $\begin{array}{c}\text { Desert and } \\
\text { hill }\end{array}$ & $1570 \sim 1710$ & 11.2 & $16 \%$ \\
\hline
\end{tabular}

\subsection{Import of geographical information data}

Geographical information data are GIS graphical data of the GIS map which serves to wire selection of the overhead line, it consists of the vector data and grid data from types, it is classified as 3 data types from category, which as basic geographical data, power special data and engineering data respectively.

Three dimensional route selection of the power transmission line starts from processing of the three dimensional geographic information data. Three dimensional geographical information data source in the engineering area is expanded by the data processing tools. According to known coordination system information of this engineering, longitudinal and latitude parameter of ground is inputted in date process setting, and then set the central longitudinal and input the corresponding coordination mode, selection of the banding method and the central longitudinal line. After the three-dimensional digital wire selection system is opened, the imported image can be positioned in the GIS three dimensional scene [6].

\subsection{Route optimization}

Take advantage of the three dimensional visualization technology based on the GIS platform in combination with the satellite image and navigation image, optimize the preliminary design route plan according to document collection and investigation at site, is shown in table 2 and table 3 . The following principles shall be considered during optimization.

Comprehensively consider construction and operation etc factors, try to construct and operate the line close to the road; Avoid the house effectively and optimize the route in the section with great demolition volume of the house; Reasonably select position of the various crossing, selection of the road, rive, power line etc crossing positions has great influence on the engineering cost, the built and on building power transmission line with crossing shall be reduced as possible, in particular to the power transmission line at high voltage class; Sufficiently consider terrain and forest covering along the line, apply 


\section{ICMMR 2016}

the tree crossing and forest plan, reduce chewing of the

forest and protect ecology environment.

Table 2. Route adjustment comparison table

\begin{tabular}{|c|c|c|c|}
\hline \multirow{2}{*}{ Route section } & Item & $\begin{array}{c}\text { Preliminary designed route } \\
\text { plan }\end{array}$ & Optimized adjustment plan \\
\hline \multirow{2}{*}{ I section } & Route length & $1.85 \mathrm{~km} \times 2$ & $1.55 \mathrm{~km} \times 2$ \\
\cline { 2 - 4 } & Traffic status & General & Better \\
\hline \multirow{2}{*}{ II section } & Route length & $6.95 \mathrm{~km} \times 2$ & $1 \times 2$ \\
\cline { 2 - 4 } & Number of angle & $3 \times 2$ & $0.65 \mathrm{~km} \times 2$ \\
\hline \multirow{2}{*}{ III section } & Route length & $0.9 \mathrm{~km} \times 2$ & $1 \times 2$ \\
\cline { 2 - 4 } & Number of angle tower & $2 \times 2$ & $0.8 \mathrm{~km} \times 2$ \\
\hline
\end{tabular}

Table 3. Application condition comparison table of power transmission tower

\begin{tabular}{|c|c|c|c|c|c|}
\hline $\begin{array}{c}\text { Comparison } \\
\text { category }\end{array}$ & $\begin{array}{c}\text { Length } \\
\mathbf{( k m )}\end{array}$ & $\begin{array}{c}\text { Base number/proportion of } \\
\text { power transmission tower(\%) }\end{array}$ & $\begin{array}{c}\text { Base number/proportion } \\
\text { of strain tower(\%) }\end{array}$ & $\begin{array}{c}\text { Kilometre } \\
\text { Consumption per } \\
\mathbf{k m}(\mathbf{t} / \mathbf{k m})\end{array}$ \\
\hline $\begin{array}{c}\text { Preliminary } \\
\text { design }\end{array}$ & 72.5 & $169 / 85.4$ & $29 / 14.6 \%$ & 2.73 & 26.85 \\
\hline $\begin{array}{c}\text { Optimization } \\
\text { design }\end{array}$ & 71.8 & $167 / 87.9$ & $23 / 12.1 \%$ & 2.65 & 26.77 \\
\hline
\end{tabular}

Table 4. Statistic table of main crossing

\begin{tabular}{|c|c|c|c|c|}
\hline No & Name of crossing object & Class & Quantity & Crossing way \\
\hline 1 & Power line & $750 \mathrm{kV}$ & $2 \times 2$ & Drill \\
\hline 2 & Power line & $110 \mathrm{kV}$ & $2 \times 4$ & Span \\
\hline 3 & Power line & $35 \mathrm{kV}$ & $2 \times 3$ & Span \\
\hline 4 & Power line & $10 \mathrm{kV}$ & $2 \times 16$ & \\
\hline 5 & Communication line & Three classes & $2 \times 23$ & \\
\hline 6 & Weak electricity line & Low voltage line & $2 \times 8$ & \\
\hline 7 & Railway & & $2 \times 2$ & Span \\
\hline 8 & National road & & $2 \times 2$ & Span \\
\hline 9 & Land road & & $2 \times 5$ & \\
\hline 10 & Chop tree & & 64 & \\
\hline 11 & House & & 15 & \\
\hline 12 & River & & 3 & \\
\hline 13 & Geological gas pipe & & $2 \times 2$ & \\
\hline
\end{tabular}

In three dimensional power transmission line sense, main crossing in the area where this engineering passes through is clearly master, which can make up disadvantage of wire selection of the power transmission line in the two dimensional terrain figures, it plays an important reference function in the tower arrangement and positioning of the line, is shown in table 4.

\section{Conclusion}

Carry out optimization of local route of the line in this engineering and length of the preliminary design line is $2 \times 72.5 \mathrm{~km}$, the length of the optimized line is $2 \times 71.8 \mathrm{~km}$, which is $2 \times 0.7 \mathrm{~km}$ shorter than original route, and 6 pieces of the angle two circuits are cancelled, length of the line passing Woods is reduced about $300 \mathrm{~m}$. In the three dimensional power transmission line scene, main crossing in the area where the line passes is clearly master, which makes up disadvantage in wire selection of 
the power transmission line in the traditional two dimensional geology graph, which plays important reference function on tower arrangement and positioning of the line. The three dimensional GIS wire selection platform makes operation efficiency of the design person to improve greatly, which is beneficial to improve design quality and guarantee implementation of engineering technical index.

\section{References}

1. Junqiang Xu, Lixing Xing, Jun Pan. Remote Sensing Technology and Application, 21 (2006)
2. Yongsheng Zhang. Phys. Remote sensing image information system (Science Press, china, 2000)

3. Yingshi Zhao. Phys. Analysis principle and method of remote sensing application (Science Press, china, 2003)

4. Shulong Zhu, Baoshan Zhu, Hongwei Wang. Phys. Remote sensing image processing and application (Science Press, china, 2006)

5. Tianhua Li, Chonggao Liao, Wunian Yang. Journal of Geotechnical Investigation \& Surveying, 12 (2006)

6. Huajun Shi, Minzhong Wan. Electric Power Survey \& Design, 12 (2004) 